\title{
Inteligencia emocional, clima organizacional y estrés ocupacional en profesionales que prestan servicios en primera infancia
}

\section{Emotional intelligence, organizational climate and occupational stress in professionals who provide services in early childhood}

\author{
María Margarita Tirado-Vides $^{1}$ (D) Lorena Cudris-Torres $^{2}(\mathbb{D})$, Miladys Paola Redondo-Marín ${ }^{3}$ y yuz Karine \\ Jiménez- Ruiz ${ }^{4}$
}

\begin{abstract}
${ }^{1}$ Fundación Universitaria del Área Andina, Valledupar, Colombia. Email: mtirado5@areandina.edu.co
${ }^{2}$ Fundación Universitaria del Área Andina, Valledupar, Colombia. Email: 1 cudris@areandina.edu.co

${ }^{3}$ Fundación Universitaria del Área Andina, Valledupar, Colombia. Email: miredonde@areandina.edu.co

${ }^{4}$ Fundación Universitaria del Área Andina, Valledupar, Colombia. Email: 1jimenez43@areandina.edu.co
\end{abstract}

Para citar este artículo: Tirado, M. M., Cudris, L., Redondo, M. P. y Jiménez, K. (2020). Inteligencia emocional, clima organizacional y estrés ocupacional en profesionales que prestan servicios en primera infancia. Clío América, 14(27), 441-453.

http://dx.doi.org/10.21676/23897848.3763

Recibido: 19 noviembre de 2019

Aceptado: 25 de enero de 2020

Publicado en línea: marzo 03 de 2020

\section{Palabras clave: \\ inteligencia \\ emocional; \\ estrés \\ laboral; \\ clima \\ organizacion \\ al; primera infancia.}

JEL: I3; M12;

M14.

\section{Keywords:}

emotional

intelligence;

work stress;

organization

al climate;

early

childhood.

\section{RESUMEN}

El objetivo del artículo es establecer la relación entre inteligencia emocional, clima organizacional y estrés ocupacional en trabajadores que prestan servicios en primera infancia. Se realizó un estudio cuantitativo de tipo correlacional de corte transversal, utilizando un muestreo no probabilístico. Para la recuperación de los datos se aplicaron los cuestionarios de estrés ocupacional contenidos en el anexo técnico de la Resolución 2646, con el cuestionario de inteligencia emocional "Trait Meta Mood Scale" (TMSS24) y la Escala de Clima Organizacional (EDCO) a 150 empleados. La información fue analizada con el paquete estadístico SPSS, con estadígrafos que permitieron establecer correlaciones teniendo en cuenta el tipo de variable y la escala de medición. Los hallazgos indican que existe correlación significativa entre el clima organizacional y el estrés laboral y, de forma sorprendente, hubo la tendencia a que las personas que mejor clima organizacional identifican son también quienes perciben mayores niveles de estrés laboral. Se concluye que es necesario realizar un programa de prevención primaria y secundaria donde se desarrollen actividades encaminadas a fortalecer la inteligencia emocional de los trabajadores y disminuir los niveles de estrés. En próximas investigaciones se deben incluir variables como evaluación de desempeño, liderazgo y trabajo en equipo.

\section{ABSTRACT}

The objective of the article is to establish the relationship between emotional intelligence, organizational climate and occupational stress in workers who provide services in early childhood. A cross-sectional correlational quantitative study was carried out, using non-probabilistic sampling. To recover the data, the occupational stress questionnaires contained in the technical annex of Resolution 2646 were applied, with the emotional intelligence questionnaire "Trait Meta Mood Scale" (TMSS24) and the Organizational Climate Scale (EDCO) to 150 employees. The information was analyzed with the SPSS statistical package, with statistics that made it possible to establish correlations taking into account the type of variable and the measurement scale. The findings indicate that there is a significant correlation between organizational climate and work stress and, surprisingly, there was a trend that the people who identify the best organizational climate are also those who perceive higher levels of work stress. It is concluded that it is necessary to carry out a primary and secondary prevention program where activities are developed aimed at strengthening the emotional intelligence of workers and reducing stress levels. In future investigations, variables such as performance evaluation, leadership and teamwork should be included. 


\section{INTRODUCCIÓN}

El componente humano en las organizaciones es un factor clave para la competitividad y para garantizar su supervivencia en los mercados (Serban y Andanut, 2014; Lin et al., 2017; Ibrahim \& AlOmari, 2020). En este sentido, la gestión de diversas variables psicosociales tiene un impacto positivo en el rendimiento y la productividad de las personas (Pedraza et al., 2015; Pedrozo y Paz, 2016; Viloria., et al., 2016a; Díaz et al., 2017; Sánchez et al., 2017; Beltrán et al., 2018; Daza et al., 2019). En este estudio se exploran las categorías de inteligencia emocional, clima organizacional y estrés ocupacional.

Para abarcar el concepto de inteligencia emocional se parte de lo expuesto por Goleman (2018), quien considera tres temáticas principales que tienen relaciones en el trabajo: "las capacidades emocionales individuales, las habilidades para trabajar en equipo y la nueva empresa organizada con inteligencia emocional" (p. 5). En este sentido, resalta las dinámicas que ocurren en las nuevas organizaciones y la necesidad de generar capacidades de adaptación por parte de las personas a través del desarrollo de competencias y valores. Además, considera que la eficacia de los líderes está relacionada con el grado de inteligencia emocional que poseen, ya que eso les permite direccionar mejor las empresas a partir de la comprensión de los sentimientos de los trabajadores.

Las personas con estas capacidades son capaces de llevar a su equipo de trabajo a los más altos niveles de producción; de lo contrario, se asume el riesgo de que los colaboradores adopten posturas apáticas y poco productivas para la empresa u organización.

Guardiola y Basurto (2015) afirman que la inteligencia emocional es un término muy importante en los últimos años para las organizaciones, ya que abarca las habilidades de las personas para identificar, valorar y compartir sus emociones con precisión; las habilidades para el acceso y generación de sentimientos a través de los pensamientos; las habilidades para la comprensión de las emociones y la generación de conocimientos, y las habilidades para la regulación de las emociones que permitan crecimientos intelectuales. Así, la ejecución de un programa de inteligencia emocional es clave, dada su contribución significativa al desarrollo y regulación de los empleados, buscando mejorar las relaciones intra y extra laborales y, sobre todo, permitir mayor productividad (Veloso et al., 2013).
Por su parte, Ramírez y Giraldo (2016) afirman que para las organizaciones es definitivo vincular a empleados que tengan adecuados niveles de estabilidad emocional -en especial, para los cargos donde se ejercen roles de dirección-, ya que cuando las personas tienen mayor conciencia sobre sus dimensiones emocionales, logran mejores relaciones con los demás y generan mayores aportes para establecer ambientes de trabajo favorables, contribuyendo a la consecución de las metas definidas por la empresa. Las personas con estabilidad emocional tributan mayores beneficios al clima organizacional y aportan de manera más asertiva en la toma de decisiones. La inteligencia emocional impacta la convivencia laboral y fomenta las relaciones sociales favorables para disminuir los niveles de estrés y riesgos psicosociales en las organizaciones.

Así mismo, Caruso y Salovey (1996, como se citó en Arcones, 2016) consideran que si no se posee un conocimiento emocional no se puede identificar con exactitud cómo una persona se siente. En el conocimiento se fundamenta la inteligencia emocional; por lo tanto, los individuos deben tener la capacidad de saber cómo se sienten y de valorar sus sentimientos de manera pertinente: así puede existir un compromiso consigo mismos y con los otros individuos. La adecuada expresión de las emociones se relaciona con las habilidades de las personas para identificar sus sentimientos: factor clave para lograr adecuadas relaciones en los contextos laborales. En coherencia con lo anterior, Cabas et al. (2017) concluyeron sobre la necesidad de desarrollar estudios en diversos contextos organizacionales para evaluar la inteligencia emocional de las personas y generar estrategias de intervención en perspectiva de su mejoramiento.

En los estudios de Iglesias y Torres (2018) y Viloria., et al. (2016b) se expone que el clima organizacional es uno de los elementos más significativos para intervenir procesos organizacionales relacionados con el cambio, la gestión y la innovación; por su derivación, tanto en los resultados como en los procesos toma gran relevancia. El contexto en el que se desenvuelven los empleados y lo que ellos perciben sobre su ambiente laboral es crucial para mantener un ritmo adecuado de su rendimiento laboral.

La generación de un clima laboral positivo contribuye al rendimiento de los empleados, ya que permite que se sientan más cómodos en sus contextos laborales y, por lo tanto, obtienen mejores respuestas en sus comportamientos productivos (Delmas y Pekovic, 2018; Yantu, 2018; Ancarani et al., 2019; Agbajeola, 2019). 
García y Duque (2013) plantean una definición más acertada de clima organizacional, en la que se enfatiza que el clima organizacional es "la percepción y apreciación de los trabajadores con relación a los aspectos estructurales (proceso y procedimientos), las relaciones entre las personas y el ambiente físico (infraestructura y elementos de trabajo)" (p. 13), resaltando incidencias en las interacciones y comportamientos de las personas, así como los impactos sobre el rendimiento del empleado y la productividad organizacional.

Por otro lado, para Osorio y Niño (2017) el estrés se relaciona con la capacidad que tienen las personas para adaptarse a las demandas que vienen del exterior, la capacidad para enfrentar tales demandas y las respuestas físicas y psicológicas que esto genera. Es un asunto que ha tomado mucha importancia en los campos organizacionales y académicos por las implicaciones que tiene sobre las personas y su salud, así como en los rendimientos laborales. Según Limonero et al. (2004), la mayoría de las personas tienen una alta probabilidad de enfrentar estrés laboral, mientras desempeñan sus funciones, debido a las exigencias externas.

Existe una relación entre los factores psicosociales y el estrés. En este sentido, los factores de riesgo psicosociales se convierten en elementos que aumentan los grados de estrés de los trabajadores como consecuencia de la existencia de amenazas a su salud física o psicológica en los contextos laborales, ocasionando el estrés laboral (Moreno, 2011).

Sin embargo, los profesionales sanitarios en general y quienes trabajan en las áreas de enfermería en particular sufren mayores demandas dada la naturaleza de sus actividades y funciones, que implican responder a las dinámicas de las personas enfermas y de sus familiares y a las mismas exigencias organizacionales, que los condicionan y enfrentan a altos niveles de estrés. Enfrentar, de manera cotidiana, todos estos factores estresantes y aspectos relativos a los niveles de dolor de los pacientes, así como la posibilidad de la muerte de estos, los puede llevar a un desgaste personal tan alto que implique consecuencias negativas para su salud.

El estrés laboral se constituye en una problemática no solamente para el trabajador que lo padece, sino también para la organización en que trabaja (Limonero et al., 2004). En una investigación realizada por Castañeda et al. (2011) sobre estrés laboral y factores psicosociales laborales en trabajadores de atención primaria, los autores encontraron que existe mayor propensión al estrés relacionado con los factores físicos de los sitios de trabajo y las interaciones sociales. En esta investigación, el nivel de carga de trabajo no se asocia como un factor estresante ni se relaciona con la salud mental de los empleados. Sin embargo, se encontró que existe una relación significativa entre los niveles de estrés laboral y la salud mental de las personas.

En este estudio se analiza la relación de la inteligencia emocional con el estrés ocupacional en individuos que prestan servicios de atención de las necesidades de la primera infancia en una fundación. Es de anotar que poseer una inteligencia emocional les permite a los trabajadores manejar de mejor forma el estrés de las ocupaciones laborales y disminuir los riesgos psicosociales asociados al trabajo.

\section{METODOLOGÍA}

Esta investigación es no experimental; se llevó a cabo mediante un enfoque cuantitativo y tiene un diseño correlacional de corte transversal. El objetivo fundamental es determinar si las variables objeto de estudio se encuentran conectadas entre sí.

\section{Muestra}

Se trabajó con una muestra de 150 empleados de la Fundación Menores del Futuro, mediante un muestreo no probabilístico por conveniencia. La investigación se llevó a cabo en los centros de trabajo de la organización, ubicados en el departamento del Cesar, en los municipios de Astrea, Chiriguaná, el Paso, Aguachica, Valledupar y Pailitas.

\section{Instrumentos}

Para esta investigación se trabajó con información de primera mano. Se aplicaron tres instrumentos de medición: el primero de ellos fue la prueba EDCO, de Acero et al. (2006), con el objetivo de identificar las percepciones que tienen las personas al interior de la organización y la organización sobre ellos. Esta prueba consta de 40 ítems y mide 8 dimensiones, que son: relaciones interpersonales, estilo de dirección, sentido de pertenencia, retribución, disponibilidad de recursos, estabilidad, claridad y coherencia en la dirección y valores colectivos.

También se usó el cuestionario de inteligencia emocional TMMS-24, de Salovey et al. (1995), para medir la inteligencia emocional. Esta prueba consta de 24 ítems y mide las dimensiones de percepción emocional, comprensión de sentimientos y regulación emocional.

Por último, se utilizó la prueba de estrés ocupacional, contenida en el anexo técnico de la Resolución 2646 de 2008, que consta de 31 ítems medidos. 


\section{Procedimiento}

La investigación se ha desarrollado de la siguiente manera: en la fase 1 se llevó a cabo una revisión teórica que permitió fundamentar las categorías del estudio; esta etapa se llamó fase conceptual. Luego, en la fase 2 se socializó el proyecto con el área de Talento Humano y el área de Seguridad y Salud en el Trabajo, de la Fundación Menores del Futuro, con el objetivo de buscar acompañamiento y apropiación del estudio; esta fase fue llamada socialización y acompañamiento. En la fase 3, denominada empírica, se aplicaron los instrumentos de medición para recoger información sobre estrés ocupacional e inteligencia emocional y se adelantó lo pertinente a la tabulación y codificación de la información. Finalmente, en la fase 4, que se llamó analítica, se procedió a realizar el análisis e interpretación de los datos para proyectar la discusión de resultados y conclusiones.

\section{Consideraciones éticas}

Para el desarrollo de la investigación se tuvieron en cuenta las disposiciones establecidas en la Resolución 8430 de 1993 del Ministerio de Salud de Colombia y la Declaración de Helsinki de 1975. En este sentido, los sujetos participantes fueron informados de los objetivos del estudio y del tratamiento académico que recibiría la información recolectada.

\section{RESULTADOS}

La presentación de los datos de esta investigación se organizó evidenciando, primero, información descriptiva de las variables evaluadas y, luego, mostrando algunas correlaciones planteadas para la interpretación de los resultados.

La sede con mayor número de trabajadores es la de Valledupar, seguida de Chiriguaná, Astrea y Aguachica. La sede Valledupar está compuesta por los centros de trabajo de los 450 años, Francisco de Paula Santander y la Nevada. Los datos evidencian que gran parte de los trabajadores viven en unión libre (38\%), seguidos de los que están casados $(22 \%)$ y de los solteros $(16 \%)$.

El $69 \%$ de los trabajadores son profesionales, el 36,6\% ocupan cargos de docentes, el $30 \%$ son auxiliares de docentes y el $14 \%$ son psicólogos; los demás empleados ocupan cargos de coordinadores, dinamizadores, nutricionistas, pedagogos, trabajadores sociales y sociólogos.

En términos de relaciones interpersonales, el $91 \%$ las considera saludables y solo un $9 \%$ considera que se deben mejorar, tal como se muestra en la Figura 1.

\section{Relaciones Interpersonales}

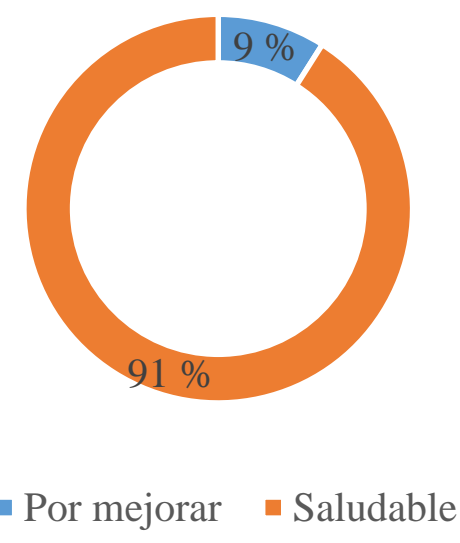

Figura 1. Relaciones Interpersonales

Fuente: elaboración propia

En cuanto a los estilos de dirección, el $83 \%$ considera que el líder genera un ecosistema de confianza entre el equipo de trabajo y apoya las decisiones del equipo; además, los trabajadores perciben que las decisiones que toma el jefe son justas y responden a una necesidad.
Los datos también permitieron evidenciar que el $76 \%$ de los trabajadores conocen los beneficios a los que tienen derecho por pertenecer a la empresa, están de acuerdo con los servicios de salud y con el salario y, además, asumen que las aspiraciones de mejoramiento y crecimiento que 
tienen en la empresa se pueden lograr. Esta información se pueda consultar en la Figura 2.

\section{Retribución}

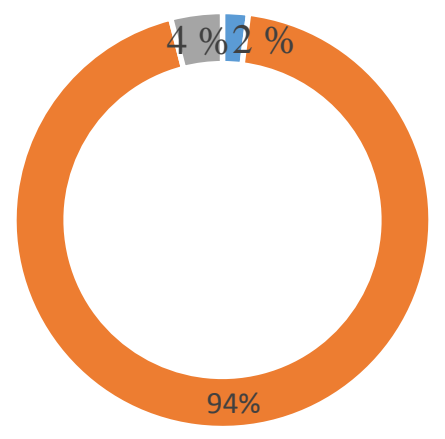

- No saludable $\quad$ Saludable $\quad$ Por mejorar

Figura 2. Retribución

Fuente: elaboración propia

Por otro lado, al $94 \%$ de los trabajadores les preocupa el futuro de la empresa, consideran que es un buen lugar para trabajar y, además, piensan que podrían recomendar esta organización a otras personas que estén buscando empleo.

En relación a la disponibilidad de recursos, el $71 \%$ de los trabajadores percibe que disponen de un espacio adecuado para trabajar y que, además, disponen de la información requerida necesaria para adelantar sus actividades laborales.

La Figura 3 presenta información sobre la estabilidad laboral de los empleados y evidencia que en la organización no se despide al personal sin justa causa y que, además, del desempeño de cada uno de los trabajadores depende la permanencia en el cargo.

\section{Estabilidad}

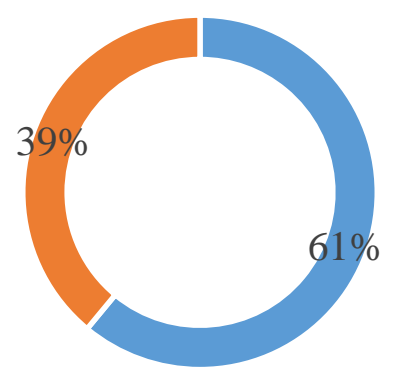

- Saludable $\quad$ - Por mejorar

Figura 3. Estabilidad

Fuente: elaboración propia

El $51 \%$ de los trabajadores comprenden claramente cuáles son los objetivos de la organización y las estrategias que actualmente está llevando a cabo para lograrlas y afirma que, además, estas son divulgadas ampliamente; sin embargo, un $42 \%$ considera que este es un factor que debe mejorar la organización y un $7 \%$ considera que no se cumple.

Por su parte, el $76 \%$ percibe que el trabajo en equipo con 
otras dependencias es bueno y que, además, cuando los trabajadores necesitan información de otras áreas esta es suministrada fácilmente y que las dificultades laborales son resueltas rápidamente en vez de estar culpando a los demás integrantes de la organización.

En general, el clima organizacional se muestra alto en el 83 $\%$ de los trabajadores; es decir, estos trabajadores perciben que las características que describe la organización son favorables.

La Figura 4 permite determinar los niveles de estrés percibido por los individuos. Se evidencia que un $66 \%$ de ellos se encuentran en un riesgo bajo y muy bajo, mientras un $6 \%$ y $7 \%$ se encuentran en riesgo alto y muy alto, lo que indica que los trabajadores que están en este rango deben ser incluidos en el programa de vigilancia epidemiológica de prevención de factores de riesgos psicosociales.

\section{Estrés Laboral}

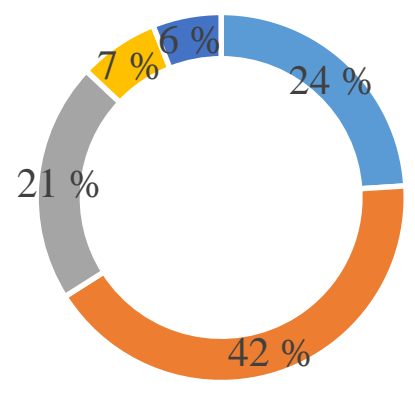

- Muy bajo $\square$ Bajo $\square$ Medio $\square$ Alto $\square$ Muy alto

Figura 4. Estrés laboral

Fuente: elaboración propia

En relación con los hallazgos de las dimensiones de inteligencia emocional, los datos evidenciaron que en la dimensión de atención el $51 \%$ de los participantes tienen niveles adecuados, mientras el $9 \%$ presentan demasiada atención y el $40 \%$ presentan poca atención: en esta dimensión, según lo indicado por los autores, puntuaciones altas y muy bajas requieren acciones de mejora (ya que esta permite identificar si la persona tiene la capacidad de sentir y expresar sus sentimientos de forma pertinente). En la dimensión de claridad, el $58 \%$ presenta un adecuado desarrollo (es decir, comprenden bien los estados emocionales) y un $42 \%$ debe mejorar. Por último, en la dimensión de reparación el $64 \%$ de los trabajadores tienen un adecuado desarrollo, siendo capaces de gestionar los estados emocionales correctamente, mientras que el $36 \%$ deben ejecutar acciones de mejora.

Tabla 1. Resultados de la prueba estadística de normalidad

\begin{tabular}{|l|l|l|l|}
\hline \multirow{2}{*}{ Variable } & \multicolumn{3}{l|}{ Kolmogorov-Smirnov } \\
\cline { 2 - 4 } & Estadístico & gl & Sig. \\
\hline EDCO total & 0,147 & 139 & 0,000 \\
\hline Relaciones Interpersonales & 0,264 & 139 & 0,000 \\
\hline
\end{tabular}

\section{Estadísticos Inferenciales}

Para la elección del método de correlación de las variables se tuvo en cuenta, en primera medida, el tipo de variable. Debido a que las categorías más bajas de las dimensiones de clima organizacional y de inteligencia emocional presentan unas frecuencias muy bajas e, incluso, sin ningún caso, no es posible realizar análisis de tablas cruzadas ni Chi2. Se optó por usar los datos cuantitativos en escala de intervalo de cada instrumento.

En primera medida, se llevó a cabo una prueba de normalidad, a través del estadístico Kolmogorov-Smirnov, paramétricas o no paramétricas. Como puede verse en la Tabla 1, las variables no tuvieron una distribución normal; por ende, se escogen como pruebas de correlación a Tau-B de Kendall y Rho de Spearman. para la decisión sobre si usar pruebas de correlación 
María Margarita Tirado-Vides, Lorena Cudris-Torres, Miladys Paola Redondo-Marín y Luz Karine Jiménez-Ruiz

\begin{tabular}{|l|l|l|l|}
\hline Estilos Dirección & 0,322 & 139 & 0,000 \\
\hline Sentido Pertenencia & 0,230 & 139 & 0,000 \\
\hline Retribución & 0,301 & 139 & 0,000 \\
\hline Disponibilidad Recursos & 0,280 & 139 & 0,000 \\
\hline Estabilidad & 0,208 & 139 & 0,000 \\
\hline Claridad Coherencia Dirección & 0,169 & 139 & 0,000 \\
\hline Valores Colectivos & 0,287 & 139 & 0,000 \\
\hline AtencionTMM24 & 0,199 & 139 & 0,000 \\
\hline ClaridadTMM24 & 0,154 & 139 & 0,000 \\
\hline ReparacionTMM24 & 0,185 & 139 & 0,000 \\
\hline Estrés General & 0,196 & 139 & 0,000 \\
\hline
\end{tabular}

Fuente: elaboración propia

La Tabla 2 muestra hallazgos de las correlaciones entre la medición de estrés, la medición de las dimensiones y el resultado global de clima organizacional. Se observa una correlación altamente significativa entre el clima organizacional y el estrés laboral en ambos estadísticos. De forma sorprendente, hubo la tendencia a que las personas que mejor clima organizacional perciben, mayor nivel de estrés laboral reportan. La misma tendencia sucedió con tres de las dimensiones del clima, puesto que retribución, disponibilidad de recursos y claridad y coherencia en la dirección también tuvieron una correlación positiva altamente significativa con la medida de estrés laboral.

Tabla 2. Correlaciones no paramétricas entre Estrés Laboral y las dimensiones y resultado global de la medición de Clima Organizacional

\begin{tabular}{|l|l|l|l|l|}
\hline & $\begin{array}{l}\text { Tau-B de } \\
\text { kendall }\end{array}$ & $\begin{array}{l}\text { Sig. } \\
\text { (bilateral) }\end{array}$ & $\begin{array}{l}\text { Rho de } \\
\text { Spearman }\end{array}$ & $\begin{array}{l}\text { Sig. } \\
\text { (bilateral) }\end{array}$ \\
\hline Clima Organizacional Global &, $218^{* *}$ & 0,000 &, $271^{* *}$ & 0,001 \\
\hline Relaciones Interpersonales & $-0,004$ & 0,955 & $-0,019$ & 0,821 \\
\hline Estilos de Dirección & 0,005 & 0,936 & 0,018 & 0,833 \\
\hline Sentido de Pertenencia & 0,031 & 0,631 & 0,036 & 0,671 \\
\hline Retribución &, $248^{* *}$ & 0,000 &, $324^{* *}$ & 0,000 \\
\hline Disponibilidad de Recursos &, $245^{* *}$ & 0,000 &, $339^{* *}$ & 0,000 \\
\hline Estabilidad &, $132^{*}$ & 0,035 & 0,156 & 0,067 \\
\hline Claridad y Coherencia en la Dirección &, $182^{* *}$ & 0,004 &, $254^{* *}$ & 0,003 \\
\hline Valores Colectivos & 0,095 & 0,137 & 0,117 & 0,170 \\
\hline
\end{tabular}

Fuente: elaboración propia

*Correlación bilateral significativa con valor $\mathrm{p}<0,05$

** Correlación bilateral significativa con valor $\mathrm{p}<0,01$

De otro lado, siendo un hallazgo sorpresivo, el grado de estrés laboral no es predecible con base en el puntaje de los participantes en las tres dimensiones de inteligencia emocional. De igual manera, dada la definición teórica de la dimensión de atención, donde tanto el nivel alto como el nivel bajo son inadecuados, se hizo cruce mediante Chi2 con los niveles de estrés, hallándose un Chi2 $=5,845$ con valor $\mathrm{p}=, 665$; es decir, esta prueba tampoco apoya la idea de que tengan relación ambas variables. 


\section{DISCUSIÓN}

Los hallazgos de la investigación dan cuenta de una población que, en relación a su perfil laboral, ocupa cargos de docentes $(36,6 \%)$ y de auxiliar de docentes (30\%). Ya algunos autores, en sus estudios, señalan que la función educadora tiene determinadas exigencias en relación al cumplimiento del plan de estudio con eficiencia y eficacia en el contexto de la educación infantil, a pesar de la escasez de elementos necesarios y de la interacción conflictiva con directivos y padres de familia; esto hace que la docencia sea considerada como una de las profesiones que generan mayor estrés (Gold y Roth, 1993, como se citó en Galvan et al., 2010).

En relación a los factores que generan estrés, las condiciones laborales, el estilo de dirección y el trabajo en equipo, los resultados de este estudio dan cuenta de la satisfacción de los empleados con estos aspectos internos de la empresa, tal y como sucedió en la investigación de Blanco et al. (2009), cuyas conclusiones muestran que en este tipo de instituciones en las que se trabaja con menores de edad hay una gran cooperación entre los empleados y administrativos, existe apoyo conjunto, cohesión grupal y comunicación fluida entre los empleados.

Así mismo, en el presente estudio el $71 \%$ de los trabajadores percibe que disponen de los recursos y de un espacio adecuado para trabajar, tal y como se comportaron estas variables intervinientes en la investigación realizada por Blanco et al. (2009), donde la mayoría de los trabajadores de su contexto de estudio piensan que sus adecuaciones a los lugares de trabajo son correctas, ya que cuentan con los recursos necesarios para realizar sus actividades.

Esas condiciones, que dan cuenta de la percepción de un clima laboral adecuado, se relacionan con los resultados de niveles bajos de estrés laboral entre los colaboradores de la fundación en la cual se realizó el estudio; el $66 \%$ de los empleados se encuentra en un riesgo bajo y muy bajo de estrés laboral. Esta relación la establecieron en su estudio Jully y Quispe (2016), cuyos resultados obtenidos demostraron una correlación significativa y positiva entre las variables estrés y clima laboral, al obtener mediante un análisis bivariado una significación de $\mathrm{p}<0,00$, para un nivel de estrés bajo en un $87,50 \%$, y el clima laboral categorizado como favorable para el 44,05 \% de los integrantes de la muestra.

En relación a la inteligencia emocional como elemento de protección frente al estrés laboral, los empleados de la institución contexto de estudio presentan un nivel adecuado de atención, claridad y reparación de sus emociones. Esto coincide con los hallazgos del estudio realizado por Acosta y Burguillos (2014), en el que se establece que las estrategias de afrontamiento más usadas por los profesores para contrarrestar el estrés laboral son el crecimiento de las personas y el centrarse en lo emocional y poder desahogarse.

Así mismo, en la investigación realizada por Cordero (2015) se busca relacionar el estrés laboral, los estresores y la inteligencia emocional (esta última, evaluada con el instrumento TMMS-24, el mismo que se utiliza en este estudio, y donde se validaron tanto fuentes como niveles de estrés); de igual forma, los resultados de inteligencia emocional que se expresan en niveles medios.

Finalmente, esta relación mínima, pero significativa, entre inteligencia emocional y estrés laboral también es evidente en el estudio realizado por Gabel et al. (2012), en el que se encuentra que los individuos con mejores niveles de inteligencia emocional tienen menores niveles de estrés laboral.

Los factores psicosociales en las organizaciones son determinantes para el buen funcionamiento de los procesos humanos y contribuyen a la competitividad.

\section{CONCLUSION}

Son numerosas las investigaciones que se han realizado sobre el estrés ocupacional, donde se evidencia que existen diferentes enfermedades clínicas y factores de riesgos psicosociales que conllevan a padecerlo, evidenciándose en problemas de aprendizaje que repercuten en las relaciones entre los individuos y en el desempeño laboral. De allí la importancia de potencializar y estimular la inteligencia emocional en los colaboradores, buscando que aprendan nuevas estrategias de afrontamiento que les permitan mejorar en su cargo y en su vida cotidiana.

Las emociones en las personas pueden influir en el rendimiento laboral. Reconocer las emociones de los demás y del equipo de trabajo en las organizaciones implica un trabajo extenso, el cual se debe desarrollar bajo parámetros de la psicología organizacional que busquen promover mecanismos que contribuyan al mejoramiento de la calidad de vida de los individuos y prevengan el origen de alteraciones patológicas y mentales.

Por otro lado, la calidad de vida en el contexto ocupacional hace referencia a las características de trabajo en una 
organización, orientadas al bienestar general y a la salud de los individuos en la ejecución de sus funciones; este es responsable del éxito de la organización en atraer, retener y desarrollar sus talentos y, así, alcanzar resultados increíbles. Por lo tanto, para una organización se vuelve necesario ofrecer un excelente ambiente laboral, dándole relevancia a la higiene, a la seguridad, y fomentando la autorrealización personal y los espacios de convivencia familiar y grupal. Las organizaciones deben reconocer que mantener una fuerza de trabajo eficiente, comprometida y motivada implica optimizar el estilo de vida y bienestar, y que, si los colaboradores se encuentran capacitados, tendrán un mayor empoderamiento en los procesos de la empresa.

Los datos de esta investigación permitieron concluir que, en general, los trabajadores que prestan servicios de atención a la primera infancia tienen un clima organizacional alto y que, además, los niveles de estrés se encuentran, en su gran mayoría, en nivel bajo y muy bajo. La variable de inteligencia emocional tuvo puntajes adecuados en las dimensiones de atención, reparación y claridad, con puntuaciones que oscilan entre $51 \%$ y $64 \%$, lo que indica la necesidad de fortalecer estas competencias en los trabajadores.

En este estudio, las personas que percibían un mejor clima organizacional fueron las que tuvieron mayor tendencia a experimentar estrés laboral. Los resultados tampoco permitieron establecer correlaciones significativas entre inteligencia emocional y estrés laboral. Las relaciones entre clima organizacional y estrés laboral se explican por las incidencias que tiene el segundo en las percepciones de los empleados sobre el ambiente de trabajo.

Para futuras investigaciones, se recomienda ampliar la muestra e incluir variables como evaluación de desempeño, liderazgo y trabajo en equipo; además, sería interesante plantear un estudio para comparar poblaciones que trabajen diferentes programas, determinando así la existencia de patrones según el tipo de población atendida.

Se sugiere a la empresa realizar un programa de prevención primaria y secundaria en el que se desarrollen actividades encaminadas a fortalecer la inteligencia emocional en sus colaboradores y disminuir los niveles de estrés, y que permita identificar los puntos fuertes y débiles de la institución para tomar decisiones de manera oportuna, en función de los resultados y logros alcanzados.

\section{Declaración sobre conflictos de interés}

Los autores se permiten declarar que no existen conflictos de intereses en el estudio y que los resultados son independientes con respecto a las instituciones financiadoras y de apoyo.

\section{REFERENCIAS BIBLIOGRÁFICAS}

Acero, Y., Echeverri, L., Lizarazo, S., Quevedo, A. y Sanabria, B. (2010). Escala de clima organizacional (edco). Fundación Universitaria Konrad Lorenz. https://es.scribd.com/document/353333365 Escala-de-Clima-Organizacional-Edco

Acosta, M. y Burgillos, A. (2014). Estrés y Burnout en profesores de primaria y secundaria de Huelva: las estrategias de afrontamiento como factor de protección. Revista INFAD de Psicología. International Journal of Developmental and Educational Psychology, $\quad$ 4(1), 303-310. https://doi.org/10.17060/ijodaep.2014.n1.v $\underline{4.616}$

Agbajeola, R. O. (2019). Organizational Climate and Teachers Characteristics as Predictors of Secondary Schools' Productivity. KIU Journal of Social Sciences, 5(1), 163-169. https://www.ijhumas.com/ojs/index.php/ki ujoss/article/view/488

Ancarani, A., Mauro, C. D. y Giammanco, M. D. (2019). Linking Organizational Climate to Work Engagement: A Study in the Healthcare Sector. International Journal of Public Administration, 42(7), 547-557. https://doi.org/10.1080/01900692.2018.149 $\underline{1595}$

Arcones, V. (2016). La Inteligencia emocional 
INTELIGENCIA EMOCIONAL, CLIMA ORGANIZACIONAL Y ESTRÉS OCUPACIONAL EN PROFESIONALES QUE PRESTAN SERVICIOS EN PRIMERA INFANCIA

en el trabajo (Tesis de maestría, Universidad de Valladolid). Repositorio institucional Uva. http://uvadoc.uva.es/handle/10324/18142

Beltrán, R. J., López, G. J. y Vélez B. O. (2018). Relación entre liderazgo e innovación en las pymes de la comuna once de Medellín. Clío América, 12(23), 52-61. https://doi.org/10.21676/23897848.2616

Blanco J., Rodríguez, P. y Díaz, P. (2009). Factores de riesgo psicosocial, autoeficacia y satisfacción laboral en trabajadores de empresas de ocio y tiempo libre. Trabajo de Grado. Universidad de La Rioja. España http://www.scielo.org.co/scielo.php?script= sci_nlinks\&ref=000142\&pid=S0121$\underline{0807201500010000700015 \& \operatorname{lng}=\mathrm{en}}$

Cabas, H. K., González, B. Y. y Hoyos, R. P. (2017). Teorías de la inteligencia y su práctica en el siglo XXI: Una revisión. Clío América, 11(22), 254-270. https://doi.org/10.21676/23897848.2445

Castañeda, H., Colunga, C., Preciado, M., Aldrete, M. y Aranda, C. (2011). Estrés organizacional y factores psicosociales laborales asociados a salud mental en trabajadores de atención primaria. Revista Waxapa, $\quad 3(5), \quad$ 80-88. https://www.medigraphic.com/cgibin/new/resumen.cgi?IDARTICULO=3292 $\underline{0}$

Cordero, A. (2015). Estrés laboral, fuentes de estrés e inteligencia emocional en los trabajadores de centro de urgencias $y$ emergencias (tesis de doctorado, Universidad de Extremadura). Base de datos https://dialnet.unirioja.es/servlet/tesis?codi $\mathrm{go}=45708$

Daza, A., Viloria, J. y Miranda, L. F. (2019). Gerencia del Talento Humano y Calidad del Servicio Educativo en Universidades Públicas de la Región Caribe de Colombia. Revista ESPACIOS, 40(44), 10-20. http://www.revistaespacios.com/a19v40n4 4/19404410.html

Delmas, M. A. y Pekovic, S. (2018). Organizational configurations for sustainability and employee productivity: A qualitative comparative analysis approach. Business \& Society, 57(1), 216251.

https://doi.org/10.1177/0007650317703648

Díaz, S. C., López, L. M. y Roncallo, L. L. (2017). Entendiendo las generaciones: una revisión del concepto, clasificación y características distintivas de los Baby Boomers, X y Millennials. Clío América, 11(22), 188-204. https://doi.org/10.21676/23897848.2440

Gabel-Shemueli, S., Peralta, V., Paiva, R. y Aguirre, G. (2012). Estrés laboral: relaciones con inteligencia emocional, factores demográficos y ocupacionales. Revista Venezolana de Gerencia (RVG), 17(58), 271-290. https://www.redalyc.org/articulo.oa?id=29 
$\underline{023348005}$

Galvan, M., Aldrete, R., Preciado, M. y Medina, S. (2010). Factores psicosociales y síndrome burnout en docentes de nivel preescolar de una zona escolar de Guadalajara, México. Revista de Educación y Desarrollo, $\quad 14(1), \quad 5-12$. http://www.cucs.udg.mx/revistas/edu_desar rollo/anteriores/14/014_Galvan.pdf

García, M. y Duque, J. (2013). Gestión humana y responsabilidad social empresarial: un enfoque estratégico para la vinculación de prácticas responsables a las organizaciones. Libre empresa, 9(1), 13-37. https://doi.org/10.18041/1657-

\section{5/libreempresa.2012v9n1.2965}

Goleman, D. (2018). La Inteligencia Emocional En la empresa. Editorial Conecta.

Guardiola, C. y Basurto, K. (2015). Inteligencia emocional y desempeño laboral de los directivos del corporativo adventista del norte de México. Apuntes Universitarios. Revista de Investigación, 5(1), 69-77. https://dialnet.unirioja.es/servlet/articulo?c $\underline{\text { odigo }=5051791}$

Ibrahim, R. y AlOmari, G. (2020). The effect of talent management on innovation: Evidence from Jordanian Banks. Management Science Letters, 10(6), 1295-1306. http://growingscience.com/beta/ms1/3596the-effect-of-talent-management-oninnovation-evidence-from-jordanianbanks.html
Chiavenato, I. (2017) Comportamiento Organizacional la dinámica del éxito en las organizaciones ( $3^{\mathrm{a}}$ ed.). Mc Graw Hill.

Iglesias, A. J. y Torres, J. (2018). Un acercamiento al clima organizacional. Revista Cubana de Enfermería, 34(1), 1224.

http://www.revenfermeria.sld.cu/index.php lenf/article/view/1257/336

Jully, S. y Quispe, G. R. (2016). Niveles de estrés y clima laboral en los colaboradores de una empresa privada de Lima (Tesis de pregrado, Universidad Autónoma del Perú). Repositorio institucional. http://repositorio.autonoma.edu.pe/handle/ $\underline{\text { AUTONOMA/366 }}$

Limonero, J., Tomás, J., Fernández, J. y Gómez, J. (2004). Influencia de la inteligencia emocional percibida en el estrés laboral de enfermería. Ansiedad y estrés, 10(1), 29-41. http://scielo.isciii.es/scielo.php?script=sci arttext\&pid=S1988-348X2019000300010

Lin, C., Yu-Ping Wang, C., Wang, C. Y. \& Jaw, B. S. (2017). The role of human capital management in organizational competitiveness. Social Behavior and Personality: an International Journal, 45(1), 81-92.

Moreno, B. (2011). Factores y riesgos laborales psicosociales: conceptualización, historia y cambios actuales. Medicina y Seguridad del trabajo, $\quad 57, \quad 4-19$. https://doi.org/10.2224/sbp.5614 
INTELIGENCIA EMOCIONAL, CLIMA ORGANIZACIONAL Y ESTRÉS OCUPACIONAL EN PROFESIONALES QUE PRESTAN SERVICIOS EN PRIMERA INFANCIA

Osorio, J. E. y Niño, L. C. (2017). Estrés laboral: estudio de revisión. Diversitas: Perspectivas en Psicología, 13(1), 91-100. https://doi.org/10.15332/s1794$\underline{9998.2017 .0001 .06}$

Pedraza, A. L., Obispo, S. K., Vásquez, G. L. y Gómez, G. L. (2015). Cultura organizacional desde la teoría de Edgar Schein: estudio fenomenológico. Clío América, 9(17), 17-25. https://doi.org/10.21676/23897848.1462

Pedrozo, R. B. y Paz, M. A. (2016). Estilos de liderazgo femenino como factor influyente en las habilidades de los equipos de trabajo en el sector Asegurador. Clío América, 10(19),

https://doi.org/10.21676/23897848.1656

Ramírez, D. A. y Giraldo, M. E. (2016). El entrenamiento en inteligencia emocional como base para disminuir los niveles de estrés laboral (Monografía de especialización. Universidad de Buenaventura, Medellín). Repositorio Institucional.

http://bibliotecadigital.usb.edu.co/handle/1 $\underline{0819 / 3369}$ ?mode=full

Salovey, P., Mayer, J. D., Goldman, S. L., Turvey, C. \& Palfai, T. P. (1995). Emotional attention, clarity, and repair: Exploring emotional intelligence using the Trait MetaMood Scale. In J. W. Pennebaker (Ed.), Emotion, disclosure, \& health (pp. 125154). American

Psychological
Association. https://doi.org/10.1037/10182$\underline{006}$

Sánchez, J., Viloria, J. y Miranda, L. (2017). Los grupos informales en las organizaciones escolares: un acercamiento desde la gestión educativa. Praxis, 13(1), 56-68. https://doi.org/10.21676/23897856.2068

Serban, A. y Andanut, M. (2014). Talent competitiveness and competitiveness through talent. Procedia Economics and Finance, $\quad 16$, 506-511. https://doi.org/10.1016/S2212-

\section{1(14)00831-4}

Veloso, C., Cuadra, A., Antezana, I., Avendaño, R. y Fuentes, L. (2013). Relación entre inteligencia emocional, satisfacción vital, felicidad subjetiva y resiliencia en funcionarios de educación especial. Estudios pedagógicos (Valdivia), $39(2)$, 355-366.

\section{https://doi.org/10.4067/S0718-}

$\underline{07052013000200022}$

Viloria, E. J, Pedraza, A. L, Cuesta, T. K. y Pérez, C. K. (2016a). Liderazgo informal en las organizaciones: reflexiones sobre su impacto e influencia en la competitividad. Clío América. 10(19), 31-42. https://revistas.unimagdalena.edu.co/index. php/clioamerica/article/view/1658/1164

Viloria, J., Pertúz, S., Daza, A. y Pedraza, L. (2016b). Aproximación al clima organizacional de una empresa promotora de salud. Revista Cubana de Salud Pública, 
María Margarita Tirado-Vides, Lorena Cudris-Torres, Miladys Paola Redondo-Marín y Luz Karine Jiménez-Ruiz 42(1). Financial Agency. Jurnal Aplikasi

http://www.revsaludpublica.sld.cu/index.ph Manajemen, $\quad$ 16(2), 330-335. p/spu/article/view/592/481 http://dx.doi.org/10.21776/ub.jam.2018.01

Yantu, I. (2018). Organizational Climate for $\underline{6.02 .16}$ Work Motivation Stimulation in Regional 